\title{
Efficient solid-state host-guest light-emitting electrochemical cells based on cationic transition metal complexes
}

\author{
Hai-Ching Su and Chung-Chih $\mathrm{Wu}^{\mathrm{a})}$ \\ Department of Electrical Engineering, National Taiwan University, Taipei, Taiwan 10617, Republic \\ of China; Graduate Institute of Electro-Optical Engineering, National Taiwan University, Taipei, Taiwan \\ 10617, Republic of China; and Graduate Institute of Electronic Engineering, National Taiwan \\ University, Taipei, Taiwan 10617, Republic of China \\ Fu-Chuan Fang and Ken-Tsung Wong \\ Department of Chemistry, National Taiwan University, Taipei, Taiwan 10617, Republic of China
}

(Received 21 October 2006; accepted 24 November 2006; published online 29 December 2006)

The authors demonstrate highly efficient solid-state light-emitting electrochemical cells (LECs) consisting of green-emitting $\left[\operatorname{Ir}(\mathrm{dFppy})_{2}(\mathrm{SB})\right]^{+}\left(\mathrm{PF}_{6}^{-}\right)$as the host and orange-emitting $\left[\operatorname{Ir}(\text { ppy })_{2}(\mathrm{SB})\right]^{+}\left(\mathrm{PF}_{6}^{-}\right)$as the guest [where dFppy is 2-(2,4-difluorophenyl)pyridine, $\mathrm{SB}$ is 4,5-diaza-9,9'-spirobifluorene, and ppy is 2-phenylpyridine]. Photophysical studies show that with the optimized host-guest compositions, the emission is mainly from the guest and photoluminescence quantum yields are largely enhanced over those of pure host and guest films due to suppressed intermolecular interactions. Correspondingly, LECs based on such host-guest cationic complex systems show substantially enhanced quantum efficiencies (power efficiencies) of up to $10.4 \%(36.8 \mathrm{~lm} / \mathrm{W})$, representing a 1.5 times enhancement compared to those of pure host and guest devices. Such results indicate that the host-guest system is essential and useful for achieving highly efficient solid-state LECs. (C) 2006 American Institute of Physics. [DOI: 10.1063/1.2425008]

Solid-state light-emitting electrochemical cells (LECs) possess several advantages over conventional organic lightemitting diodes (OLEDs). In LECs, electrochemically doped regions induced by spatially separated ions under a bias form Ohmic contacts with electrodes, giving balanced carrier injection, low operating voltages, and consequently high power efficiencies. ${ }^{1,2}$ As such, LECs generally require only a single emissive layer, which can be easily processed from solutions and can conveniently use air-stable electrodes, while OLEDs typically require more sophisticated multilayer structures and low-work-function cathodes. ${ }^{3,4}$ Compared with conventional polymer LECs that are usually composed of an emissive conjugated polymer, a salt and an ion-conducting polymer, ${ }^{1,2}$ LECs based on cationic transition metal complexes show several further advantages and have attracted much attention in recent years. ${ }^{5-16}$ In such devices, no ion-conducting material is needed since these metal complexes are intrinsically ionic. Furthermore, higher electroluminescent (EL) efficiencies are expected due to the phosphorescent nature of the metal complexes.

In general, LECs are composed of neat films of emissive materials, which very often suffer self-quenching induced by interactions between closely packed molecules. Many efforts have been made to enhance external quantum efficiencies (EQEs) of LECs based on cationic metal complexes by reducing self-quenching of the emissive materials. Modifying the molecular structures, such as adding bulky substituents on ligands ${ }^{8}$ or utilizing bulky auxiliary ligands, ${ }^{14}$ had been shown to suppress interchromophore interaction to some degree, improving EQEs of LECs. Recently, we had reported highly efficient green and orange LECs (peak EQE > 7\% photon/electron) using neat films of cationic iridium com-

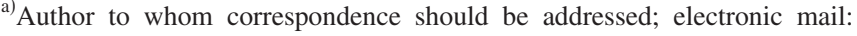
chungwu@cc.ee.ntu.edu.tw plexes having the bulky spirobifluorene-based auxiliary ligand for superior steric hindrance. ${ }^{14}$

Since these newly developed cationic complexes are intrinsically efficient, to further reduce self-quenching and increase EL efficiency, one possible approach is to spatially disperse the emitting complex (guest) into a matrix complex (host), as previously reported for conventional OLEDs and solid-state LECs. ${ }^{4,16,17}$ In this letter, we investigate the photoluminescent (PL) and EL properties of the host-guest cationic complex system using our newly developed orangeemitting Ir complex as the guest and the green-emitting Ir complex as the host. Results show that with the optimized host-guest composition, both PL and EL efficiencies can be enhanced by about $50 \%$, giving a peak EQE over $10 \%$, which is among the highest reported for solid-state LECs.

Molecular structures of the host $\left[\operatorname{Ir}(\mathrm{dFppy})_{2}(\mathrm{SB})\right]^{+}\left(\mathrm{PF}_{6}^{-}\right)$ and the guest $\left[\operatorname{Ir}(\mathrm{ppy})_{2}(\mathrm{SB})\right]^{+}\left(\mathrm{PF}_{6}^{-}\right)$are shown in Fig. 1 [where dFppy is 2-(2,4-difluorophenyl)pyridine, SB is 4,5diaza- $9,9^{\prime}$-spirobifluorene, and ppy is 2-phenylpyridine]. ${ }^{14}$ Neat films of the guest exhibit orange PL while those of the host emit in green due to the fluoro substituents on the ppy ligands. ${ }^{14}$ Both show relatively high PL quantum yields (PLQYs) ( $\sim 31 \%$ for both) even in neat films due to superior steric hindrance provided by the SB ligand. ${ }^{14}$ The mixed host-guest films $(\sim 100 \mathrm{~nm})$ for PL studies were spin coated

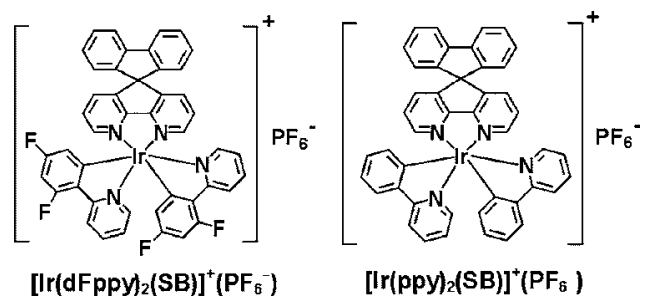

FIG. 1. Molecular structures of the host molecule $\left[\operatorname{Ir}(\mathrm{dFppy})_{2}(\mathrm{SB})\right]^{+}\left(\mathrm{PF}_{6}^{-}\right)$ and the guest molecule $\left[\operatorname{Ir}(\mathrm{ppy})_{2}(\mathrm{SB})\right]^{+}\left(\mathrm{PF}_{6}^{-}\right)$. 

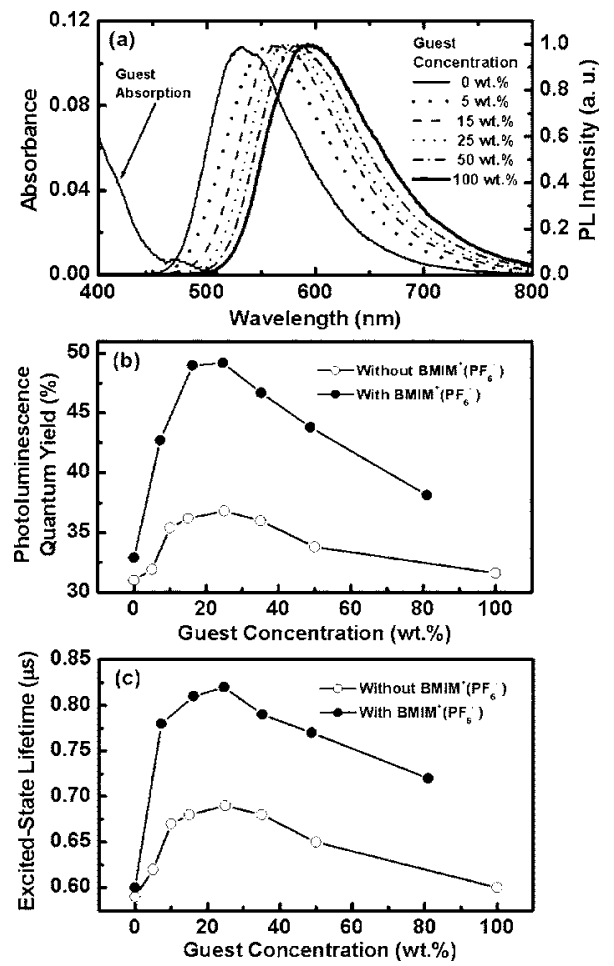

FIG. 2. (a) Absorption spectrum of the neat guest film and PL spectra of host-guest films with various guest concentrations [without $\mathrm{BMIM}^{+}\left(\mathrm{PF}_{6}^{-}\right)$], (b) photoluminescence quantum yields, and (c) excited-state lifetimes as a function of the guest concentration for host-guest films without and with $\mathrm{BMIM}^{+}\left(\mathrm{PF}_{6}^{-}\right)(19 \mathrm{wt} \%)$.

onto quartz substrates using mixed solutions of various ratios. Since in LECs, a salt $\mathrm{BMIM}^{+}\left(\mathrm{PF}_{6}^{-}\right)$of $19 \mathrm{wt} \%$ (where BMIM is 1-butyl-3-methylimidazolium) was also added to provide additional mobile ions and to shorten the device response time, ${ }^{15}$ PL properties of the host-guest-salt threecomponent system were also characterized. PL spectra of thin-film samples were measured using the $325 \mathrm{~nm}$ line of the $\mathrm{He}-\mathrm{Cd}$ laser as the excitation and charge coupled device (CCD) spectrograph, while PLQYs were measured using a calibrated integrating sphere coupled with a cooled CCD spectrograph. ${ }^{14}$ Excited-state lifetimes of the samples were measured using the time-correlated single-photon counting technique ${ }^{14}$ LEC devices were fabricated by spin coating the mixed solutions of host, guest, and $\mathrm{BMIM}^{+}\left(\mathrm{PF}_{6}^{-}\right)(19 \mathrm{wt} \%)$ on indium tin oxide coated glass substrates to form an $\sim 100 \mathrm{~nm}$-thick film, followed by thermal evaporation of a $150 \mathrm{~nm}$ Ag top contact (cathode). The electrical and emission characteristics of LECs were measured under constant bias voltages.

Figure 2(a) shows the absorption spectrum of the guest and the PL spectra of the host-guest two-component systems having various guest concentrations. With the increase of the guest concentration, a gradual redshift from the hostlike emission to the guestlike emission is observed. As shown in Fig. 2(b), the highest PLQY of $\sim 37 \%$ (vs $\sim 31 \%$ of neat host and guest films) is obtained at the guest concentration of 25 wt \%, at which the emission is near complete from the guest. Accompanying this enhanced PLQY is the longer excited-state lifetime $(0.69 \mu \mathrm{s})$ compared to those of neat films $(0.59 \mu$ s for the host and $0.60 \mu$ s for the guest), indicating the effectiveness of the dispersion in suppressing quenching mechanisms of guest molecules. Less complete transfer is observed at lower guest concentrations, which Downloaded 17 Feb 2009 to 140.112 .113 .225 . Redistribution subject
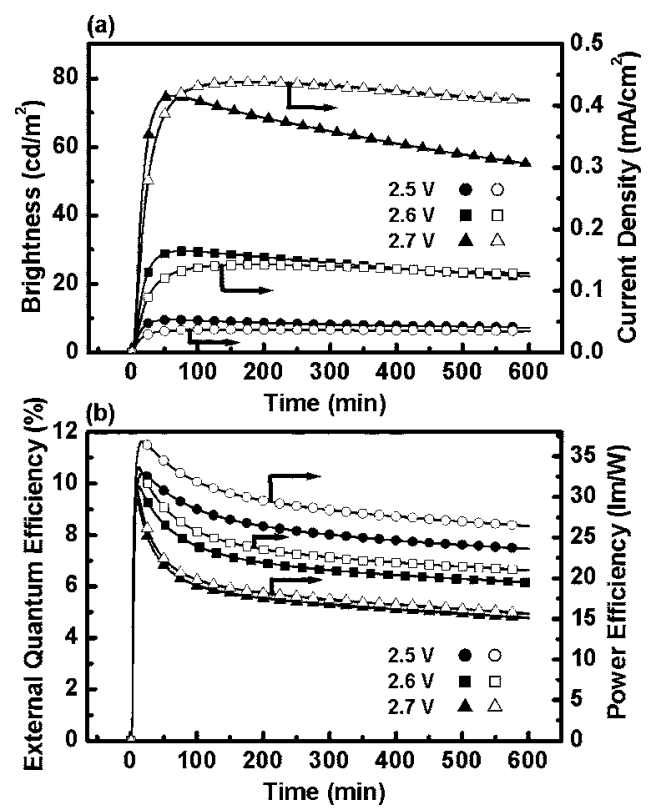

FIG. 3. (a) Brightness (solid symbols) and current density (open symbols) and (b) external quantum efficiency (solid symbols) and power efficiency (open symbols) as a function of time under a constant bias voltage of 2.5-2.7 V for the host-guest LEC with host, guest, and $\mathrm{BMIM}^{+}\left(\mathrm{PF}_{6}^{-}\right)$concentrations of 56,25 , and $19 \mathrm{wt} \%$, respectively.

may be associated with the less extensive overlap between the host emission and the weak guest absorption. A calculation of the Förster radius for the host-guest energy transfer gives a small value of $<16 \AA{ }^{18}$ indicating inefficient energy transfer at lower guest concentrations. With the addition of $\mathrm{BMIM}^{+}\left(\mathrm{PF}_{6}^{-}\right)(19 \mathrm{wt} \%)$, the trend in PL properties (spectra (not shown), PLQYs [Fig. 2(b)], and lifetimes [Fig. 2(c)]) as a function of the guest concentration is similar to those of the two-component system. Yet, an even higher PLQY of $\sim 50 \%$ (about 1.6 times enhancement compared to PLQYs of neat host and guest films) and longer excited-state lifetime of $\sim 0.82 \mu \mathrm{s}$ are observed around the guest concentration of $25 \mathrm{wt} \%$. It appears that $\mathrm{BMIM}^{+}\left(\mathrm{PF}_{6}^{-}\right)$not only provides additional mobile ions but is also effective in suppressing interchromophore quenching.

Figure 3(a) shows the time-dependent brightness and current density under constant biases of $2.5-2.7 \mathrm{~V}$ for the LEC using the mixture giving the highest PLQY [i.e., with host, guest, and $\mathrm{BMIM}^{+}\left(\mathrm{PF}_{6}^{-}\right)$concentrations of 56,25 , and 19 wt \%, respectively]. After the bias was applied, the current first increased and then stayed rather constant. On the other hand, the brightness first increased with the current and reached the maxima of 10,30 , and $75 \mathrm{~cd} / \mathrm{m}^{2}$ at $<1 \mathrm{~h}$ under biases of $2.5,2.6$, and $2.7 \mathrm{~V}$, respectively. The brightness then dropped with time with a rate significantly depending on the bias voltage (or current). ${ }^{15}$ Corresponding timedependent EQEs and power efficiencies of the same device are shown in Fig. 3(b). When a forward bias was just applied, the EQE was rather low due to poor carrier injection. During the formation of the $p$ - and $n$-type regions near electrodes, the capability of carrier injection was improved and the EQE thus rised rapidly. The peak EQE, cd/A, and peak power efficiencies at 2.5, 2.6, and $2.7 \mathrm{~V}$ are $(10.4 \%$, $29.3 \mathrm{~cd} / \mathrm{A}$, and $36.8 \mathrm{~lm} / \mathrm{W}),(9.9 \%, 27.9 \mathrm{~cd} / \mathrm{A}$, and $33.7 \mathrm{~lm} / \mathrm{A})$, and $(9.4 \%, 26.5 \mathrm{~cd} / \mathrm{A}$, and $30.8 \mathrm{~lm} / \mathrm{W})$, respectively. The drop of efficiencies and brightnesses after reaching the peak value, as commonly seen in solid-state to AlP license or copyright; see http://apl.aip.org/apl/copyright.jsp 

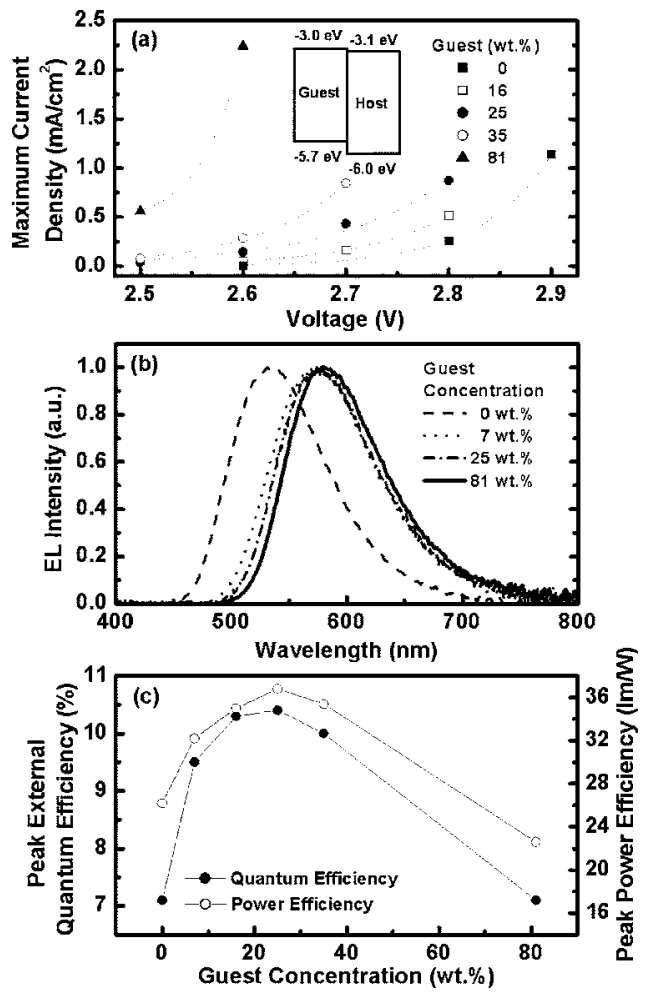

FIG. 4. (a) Maximum current density vs voltage characteristics for LECs with various guest concentrations. (b) EL spectra (at $2.8 \mathrm{~V}$ ) for LECs with various guest concentrations. (c) Peak external quantum efficiencies and peak power efficiencies (at current densities $<0.1 \mathrm{~mA} / \mathrm{cm}^{2}$ ) of host-guest LECs as a function of the guest concentration. Inset of (a): the energy level diagram of the host and guest molecules.

LECs, ${ }^{5-16}$ may be associated with a few factors. Before the current reaches a steady value, the carrier recombination zone may keep moving closer to one electrode due to discrepancy in electron and hole mobilities, which would induce exciton quenching. Further, the decrease in brightnesses/efficiencies under a constant bias was irreversible and thus may be rationally associated with the degradation of the emissive material during the LEC operation. ${ }^{19}$

The maximum current density versus voltage characteristics of LECs with various guest concentrations are shown in Fig. 4(a). The bias voltage required for same current drops as the guest concentration is raised. This may be understood by the energy level diagram obtained from cyclic voltammetry $^{14}$ [inset of Fig. 4(a)]. Such an energy level alignment favors carrier injection/transport (at least for holes) through the smaller-gap guest and direct carrier recombination/exciton formation on the guest (rather than host-guest energy transfer) if the guest concentration is high enough. EL spectra of host-guest LECs with various guest concentrations [Fig. 4(b)] indeed support the mechanism of direct exciton formation on guest molecules, which would greatly reduce host emission due to incomplete energy transfer. Compared with PL spectra (e.g., Fig. 2), EL spectra are much less dependent on the guest concentration. Even with a guest concentration as low as $7 \mathrm{wt} \%$, the EL spectrum is almost the same as that of the pure guest device [i.e., the LEC with 81 wt $\%$ of guest and 19 wt $\%$ of $\left.\mathrm{BMIM}^{+}\left(\mathrm{PF}_{6}^{-}\right)\right]$, indicating predominant guest emission.

Figure 4(c) shows peak EQEs and peak power efficiencies for host-guest LECs as a function of the guest concentration (at current densities $<0.1 \mathrm{~mA} / \mathrm{cm}^{2}$ ). The peak EQEs and power efficiencies roughly follow the trend of the Downloaded 17 Feb 2009 to 140.112 .113 .225 . Redistribution subject
PLQYs. The highest peak EQE and power efficiency of $10.4 \%$ and $36.8 \mathrm{~lm} / \mathrm{W}$ are achieved at the guest concentration of $25 \mathrm{wt} \%$, coincident with the concentration giving the highest PL efficiency. Such an EQE represents a 1.5 times enhancement compared to those of pure host and guest devices. It is also worth noting that such a peak EQE approximately approaches the upper limit that one would expect from the PLQY of the host-guest emissive layer $(\sim 50 \%)$, when considering an optical outcoupling efficiency of $\sim 20 \%$ from a typical layered light-emitting device structure. Such EQEs and power efficiencies also are among the highest reported for solid-state LECs based on cationic transition metal complexes. Such results indicate that the host-guest system is essential and useful for achieving highly efficient solid-state LECs.

In summary, we have investigated photophysical and electroluminescent properties of a host-guest system consisting of the newly developed and intrinsically efficient cationic metal complexes $\left[\operatorname{Ir}(\mathrm{dFppy})_{2}(\mathrm{SB})\right]^{+}\left(\mathrm{PF}_{6}^{-}\right)$as the host and $\left[\operatorname{Ir}(\mathrm{ppy})_{2}(\mathrm{SB})\right]^{+}\left(\mathrm{PF}_{6}^{-}\right)$as the guest. Photophysical studies show that with the optimized host-guest compositions, the emission is mainly from the guest and PL quantum yields are largely enhanced over those of pure host and guest films due to suppressed intermolecular interactions. Correspondingly, host-guest LECs show much enhanced quantum efficiencies (power efficiencies) of up to $10.4 \%(36.8 \mathrm{~lm} / \mathrm{W})$, representing a 1.5 times enhancement compared to those of pure host and guest devices. Interestingly, the EL efficiency achieved with the present host-guest cationic complex system is substantially higher than those of previously reported host-guest cationic complex systems ${ }^{16}$ most likely due to higher intrinsic PL efficiencies of compounds used in this work.

${ }^{1}$ Q. Pei, G. Yu, C. Zhang, Y. Yang, and A. J. Heeger, Science 269, 1086 (1995).

${ }^{2}$ Q. Pei, Y. Yang, G. Yu, C. Zhang, and A. J. Heeger, J. Am. Chem. Soc. 118, 3922 (1996).

${ }^{3}$ C. W. Tang and S. A. VanSlyke, Appl. Phys. Lett. 51, 913 (1987).

${ }^{4}$ C. W. Tang, S. A. VanSlyke, and C. H. Chen, Appl. Phys. Lett. 65, 3610 (1989).

${ }^{5}$ J. K. Lee, D. S. Yoo, E. S. Handy, and M. F. Rubner, Appl. Phys. Lett. 69, 1686 (1996)

${ }^{6}$ E. S. Handy, A. J. Pal, and M. F. Rubner, J. Am. Chem. Soc. 121, 3525 (1999).

${ }^{7}$ C. Y. Liu and A. J. Bard, J. Am. Chem. Soc. 124, 4190 (2002).

${ }^{8}$ H. Rudmann, S. Shimada, and M. F. Rubner, J. Am. Chem. Soc. 124, 4918 (2002).

${ }^{9}$ C. Y. Liu and A. J. Bard, Appl. Phys. Lett. 87, 061110 (2005).

${ }^{10}$ J. Slinker, D. Bernards, P. L. Houston, H. D. Abruña, S. Bernhard, and G. G. Malliaras, Chem. Commun. (Cambridge) 2392 (2003).

${ }^{11}$ J. D. Slinker, C. Y. Koh, G. G. Malliaras, M. S. Lowry, and S. Bernhard, Appl. Phys. Lett. 86, 173506 (2005).

${ }^{12}$ M. S. Lowry, J. I. Goldsmith, J. D. Slinker, R. Rohl, R. A. Pascal, Jr., G. G. Malliaras, and S. Bernhard, Chem. Mater. 17, 5712 (2005).

${ }^{13}$ A. B. Tamayo, S. Garon, T. Sajoto, P. I. Djurovich, I. M. Tsyba, R. Bau, and M. E. Thompson, Inorg. Chem. 44, 8723 (2005).

${ }^{14}$ H.-C. Su, F.-C. Fang, T.-Y. Hwu, H.-H. Hsieh, H.-F. Chen, G.-H. Lee, S.-M. Peng, K.-T. Wong, and C.-C. Wu, Adv. Funct. Mater. (to be published).

${ }^{15}$ S. T. Parker, J. D. Slinker, M. S. Lowry, M. P. Cox, S. Bernhard, and G. G. Malliaras, Chem. Mater. 17, 3187 (2005).

${ }^{16}$ A. R. Hosseini, C. Y. Koh, J. D. Slinker, S. Flores-Torres, H. D. Abruña, and G. G. Malliaras, Chem. Mater. 17, 6114 (2005).

${ }^{17}$ F.-C. Chen, Y. Yang, and Q. Pei, Appl. Phys. Lett. 81, 4278 (2002).

${ }^{18}$ Modern Quantum Chemistry, edited by O. Sinanoğlu (Academic, New York, 1965), Pt. 3, p. 135.

${ }^{19}$ G. Kalyuzhny, M. Buda, J. McNeill, P. Barbara, and A. J. Bard, J. Am. Chem. Soc. 125, 6272 (2003).

AIP license or copyright; see http://apl.aip.org/apl/copyright.jsp 